\title{
Neuronal Nicotinic Synapse Assembly Requires the Adenomatous Polyposis Coli Tumor Suppressor Protein
}

\author{
Murali Krishna Temburni, ${ }^{\star}$ Madelaine M. Rosenberg, ${ }^{\star}$ Narendra Pathak, Russell McConnell, and Michele H. Jacob \\ Department of Neuroscience, Tufts University School of Medicine, Boston, Massachusetts 02111
}

\begin{abstract}
Normal cognitive and autonomic functions require nicotinic synaptic signaling. Despite the physiological importance of these synapses, little is known about molecular mechanisms that direct their assembly during development. We show here that the tumor-suppressor protein adenomatous polyposis coli (APC) functions in localizing $\alpha 3$-nicotinic acetylcholine receptors (nAChRs) to neuronal postsynaptic sites. Our quantitative confocal microscopy studies indicate that APC is selectively enriched at cholinergic synapses; APC surface clusters are juxtaposed to synaptic vesicle clusters and colocalize with $\alpha 3$-nAChRs but not with the neighboring synaptic glycine receptors or perisynaptic $\alpha 7$-nAChRs on chick ciliary ganglion (CG) neurons. We identify PSD (postsynaptic density)-93, $\beta$-catenin, and microtubule end binding protein EB1 as APC binding partners. PSD-93 and $\beta$-catenin are also enriched at $\alpha 3$-nAChR postsynaptic sites. EB1 shows close proximity to and partial overlap with $\alpha 3$-nAChR and APC surface clusters. We tested the role of APC in neuronal nicotinic synapse assembly by using retroviral-mediated in vivo overexpression of an APC dominant-negative (APC-dn) peptide to block the interaction of endogenous APC with both EB1 and PSD-93 during synapse formation in CG neurons. The overexpressed APC-dn led to dramatic decreases in $\alpha 3$-nAChR surface levels and clusters. Effects were specific to $\alpha 3$-nAChR postsynaptic sites; synaptic glycine receptor and perisynaptic $\alpha 7$-nAChR clusters were not altered. In addition, APC-dn also reduced surface membrane-associated clusters of PSD-93 and EB1. The results show that APC plays a key role in organizing excitatory cholinergic postsynaptic specializations in CG neurons. We identify APC as the first nonreceptor protein to function in localizing nAChRs to neuronal synapses in vivo.
\end{abstract}

Key words: nicotinic; cholinergic; nAChR; synapse formation; targeting; neuron; ciliary ganglion; adenomatous polyposis coli; APC

\section{Introduction}

Nicotinic acetylcholine receptors (nAChRs) function at key interneuronal synapses. Their activation mediates excitatory transmission, reinforces nicotine addiction, and increases memory formation. Malfunction of cholinergic synapses has been implicated in Alzheimer's disease, schizophrenia, nocturnal frontal lobe epilepsy, and autoimmune autonomic neuropathies.

Despite the physiological importance of these synapses, little is known about molecular mechanisms that direct their assembly. Only two proteins have been identified that organize neuronal nicotinic synapses. First, one nAChR subunit, $\alpha 3$, is essential for targeting heteromeric $\alpha 3$-containing nAChRs to the postsynaptic membrane in autonomic neurons (Williams et al., 1998). Second, PSD (postsynaptic density)-93 regulates synaptic stability; $\alpha 3-$ nAChR clusters disassemble faster after denervation in PSD-93deficient versus wild-type autonomic ganglia (Parker et al., 2004).

\footnotetext{
Received 0ct. 15, 2003; revised June 11, 2004; accepted June 11, 2004.

This work was supported by National Institutes of Health Grants NS21725 to M.H.J., DK34928 to the Gastroenterology Research on Absorptive and Secretory Processes Center, and P30 NS47243 to the Center for Neuroscience Research Cores. We thank Monica Giovanni and Howard Chen for expert technical assistance with quantitative immunofluorescence microscopy studies and yeast two-hybrid screens; Sean Sullivan for participating in preliminary studies of APC immunolocalization; and Kathleen Dunlap, Daniel Jay, and Angeles Ribera for insightful comments on this manuscript.

${ }^{*}$ M.K.T. and M.M.R. contributed equally to this work.

Correspondence should be addressed to Dr. Michele Jacob, Department of Neuroscience, Tufts University School of Medicine, 136 Harrison Avenue, Boston, MA 02111. E-mail: michele.jacob@tufts.edu.

DOI:10.1523/JNEUROSCI.1826-04.2004

Copyright $\odot 2004$ Society for Neuroscience $\quad$ 0270-6474/04/246776-09\$15.00/0
}

In addition, PSD-93 and PSD-95 couple receptor activation to downstream signaling cascades that regulate plasticity at nicotinic and glutamatergic synapses (Migaud et al., 1998; Conroy et al., 2003).

Because PSD-93 and PSD-95 organize both nicotinic and glutamatergic synapses, it is possible that a common core of proteins may organize diverse synaptic complexes. We propose that adenomatous polyposis coli (APC) may be one of these key organizers. APC is known best as a tumor-suppressor protein: lossof-function mutations cause colorectal cancer (Livingston, 2001). Although the function of APC in neurons has yet to be defined, several lines of evidence suggest that it may play a role in synaptic differentiation. Muscle-type nAChR clustering induced by agrin requires APC interactions in cultured myotubes (Wang et al., 2003). APC localizes to glutamatergic synapses in hippocampal neurons and binds PSD-95 (Matsumine et al., 1996; Yanai et al., 2000). Moreover, APC is expressed in multiple neuron types and binds $\beta$-catenin, a widely expressed adhesion complex component of cadherin-mediated synapses, suggesting APC may have a broader synaptic distribution (Brakeman et al., 1999; Fearnhead et al., 2001). An essential role of APC in neurons is indicated by the correlation of APC gene deletions with human mental retardation (Raedle et al., 2001).

We tested for a role of APC in neuronal synaptic assembly in vivo using the chick parasympathetic ciliary ganglion (CG). In these experimentally tractable neurons, excitatory cholinergic synapses lie adjacent to inhibitory glycinergic synapses (Tsen et 
al., 2000). Single CG neurons express two nAChR types, $\alpha 3$ nAChRs and $\alpha 7$-nAChRs, and glycine receptors (GlyRs) that predominantly segregate to distinct synapse-associated surface membrane microregions. The $\alpha 3$-nAChRs are concentrated in postsynaptic membrane regions that oppose presynaptic terminal active zones (Jacob et al., 1986; Loring and Zigmond, 1987). Inhibitory GlyR clusters are present in separate but proximal postsynaptic membrane microregions, all under one presynaptic terminal (Tsen et al., 2000). In contrast, $\alpha 7$-nAChRs are excluded from the synapse and localize perisynaptically on somatic spines (Jacob and Berg, 1983; Shoop et al., 1999). The discrete localization of the different receptor types provides a valuable comparison for establishing the specificity of protein interactions responsible for orchestrating cholinergic synapse assembly.

We show here that APC interactions are required for highdensity accumulations of $\alpha 3$-nAChRs, but not GlyRs, at postsynaptic sites in CG neurons in vivo. Our data identify APC as a key nicotinic cholinergic synapse-organizer and define a neural function for APC.

\section{Materials and Methods}

Antibodies. Primary antibodies used for immunolabeling (1:100 dilution) were: rabbit C-20 to APC C terminus (Santa Cruz Biotechnology, Santa Cruz, CA), mouse ab58 to APC N terminus (Abcam, Cambridge, UK), anti-Chapsyn-110/PSD-93 antiserum (Alomone Labs, Jerusalem, Israel), anti-end binding protein 1 (EB1) monoclonal antibody (mAb) (Transduction Laboratories, Lexington, KY), rabbit anti-EB1 (H-70; Santa Cruz Biotechnology), anti- $\beta$-catenin $\mathrm{mAb}$ and anti-N-cadherin mAb (Zymed Laboratories, San Francisco, CA), rat mAb35 to $\alpha 3-$ nAChRs (Developmental Studies Hybridoma Bank, Iowa City, IA), biotinylated $\alpha$-bungarotoxin (Molecular Probes, Eugene, OR) to detect $\alpha 7$ nAChRs, mAb2b to GlyRs and mAb7a to gephyrin (Alexis Biochemicals, San Diego, CA), anti-synaptic vesicle 2 (SV2) mAb (Developmental Studies Hybridoma Bank), and pan-PSD-95 family mAb (clone K28/ 86.2; Upstate Biotechnology, Lake Placid, NY). Rat anti-hemagglutinin (HA) mAb (clone 3F10; Roche Diagnostics, Indianapolis, IN) and anti-HA Y-11 antisera (Santa Cruz Biotechnology) were used for detecting HA-tagged APC dominant-negative (APC-dn) protein. Secondary reagents used at 1:1000-2000 dilution were as follows: Alexa 488- and 594-conjugated secondary antibodies raised in rabbit, rat, and mouse (affinity purified) (Molecular Probes), cyanine 3-conjugated streptavidin (Jackson ImmunoResearch, West Grove, PA), and FITC-conjugated avidin distinct cell sorter grade (Vector Laboratories, Burlingame, CA).

Ciliary ganglion dissociation. White Leghorn embryonated chick eggs were obtained from Charles River Spafas (North Franklin, CT) and maintained at $37^{\circ} \mathrm{C}$ in a forced air-draft humidified incubator until use. Embryos were staged according to the classification scheme by Hamburger and Hamilton (1951), and the days of embryonic development refer to the stage (st) rather than the actual days of incubation. The developmental stages used in this study include the following: embryonic day 7 (E7) (st 31-32), E11-E13 (st 37-39), and E18 (st 44). CGs at E11-E13 and E18 were acutely dissociated in $\mathrm{Ca}^{2+}$-free avian basal salt solution using collagenase $(1 \mathrm{mg} / \mathrm{ml}$; Type A; Roche) (Williams et al., 1998). Cells were plated onto poly-D-lysine and laminin-coated chamber slides (BD Labware, Bedford, MA) and allowed to attach to the substratum $\left(30 \mathrm{~min}\right.$ at $\left.37^{\circ} \mathrm{C}\right)$ before immunolabeling.

Quantitative confocal microscopy. Double-labeling immunofluorescence and confocal microscopy were done as described with minor modifications (Tsen et al., 2000). Surface nAChRs on freshly dissociated intact CG neurons were immunolabeled at $4^{\circ} \mathrm{C}$ for $1 \mathrm{hr}$ to avoid staining the large internal biosynthetic pool. Next, cells were rinsed with cold basal salt solution, fixed ( $1 \%$ paraformaldehyde for $5 \mathrm{~min}$ ), and permeabilized $(0.1 \%$ saponin for $2 \mathrm{~min}$ ) before labeling with other antibodies (to APC, PSD-93, $\beta$-catenin, EB1, N-cadherin, gephyrin, or HA) followed by fluorescent-conjugated secondary reagents. Controls for specific binding in double-labeling studies included omitting the first or second primary antibody in separate tests; only background labeling was detected (data not shown). Cells were examined by confocal microscopy with a Leica (Heidelberg, Germany) TCS SP2 microscope equipped with HeNe (633 $\mathrm{nm}), \operatorname{Kr}(568 \mathrm{~nm})$, and $\mathrm{Ar}(488 \mathrm{~nm})$ laser sources, using a $63 \times 1.32$ numerical aperture lens. Optical sections were acquired from the top to the bottom of each neuron at $\sim 0.5 \mu \mathrm{m}$ step intervals. Laser intensity and photomultiplier tube gain were kept constant across experiments. Settings were chosen such that pixel intensities fell below saturation levels. In addition, the wavelengths of light collected in each detection channel were set such that no detectable bleed-through occurred between the different channels. Pixel intensity profiles along randomly chosen $\sim 3$ $\mu \mathrm{m}$ segments of labeled neuronal surface membrane were assessed using Leica imaging software. Pearson's correlation coefficients $(r)$ were used to quantify the extent of overlap between two immunolabels and were calculated using Leica, Scion Image (Scion, Frederick, MD), and Microsoft Excel (Microsoft, Seattle, WA) software. Pearson's correlation coefficient values represent the mean \pm SEM of calculations from two to three different labeled surface membrane areas per cell for four to six neurons for each immunolabel pair.

Light microscopy. For some experiments, APC-dn infected and uninfected age-matched control CGs (E7, E11-E13, and E18) were processed in parallel for frozen sectioning and immunolabeling as described previously (Tsen et al., 2000). By E8, all CG neurons were functionally innervated. To detect surface $\alpha 3$-nAChRs, freshly dissected CGs were treated with collagenase $(1 \mathrm{mg} / \mathrm{ml} ; 10 \mathrm{~min})$ to increase reagent access and incubated with $\mathrm{mAb} 35$ at $4^{\circ} \mathrm{C}$. CGs were rinsed in cold PBS, fixed in $1 \%$ paraformaldehyde for $30 \mathrm{~min}$, and processed for frozen sectioning ( $8-10$ $\mu \mathrm{m}$ thick cryosections). Sections were labeled with other antibodies (to HA, gephyrin, APC, PSD-93, or N-cadherin), examined with a Zeiss Axioscope (Zeiss, Thornwood, NY) microscope, and photographed with a SPOT color CCD camera and software (Diagnostic Instruments, Sterling Heights, MI). For all experiments, images were acquired using identical settings for gain and exposure time such that pixel intensities were below saturation levels. To quantify changes in surface membraneassociated labeling in APC-dn versus uninfected control neurons, we assessed pixel intensity profiles along $\sim 3 \mu \mathrm{m}$ length segments of the surface membrane using Scion Image. For each immunolabel, pixel intensities for three or more segments per neuron and a total of four to six neurons were evaluated for control and APC-dn expressing CGs. The pixel intensities of the sampled membrane segments were then binned into incremental groups of 10 pixel intensity steps (e.g., $0-9,10-19 . \ldots$, up to 255 saturation). We then calculated the percentage of pixels that belonged to each pixel intensity category and plotted the relative frequency distributions of pixel intensities (data shown as graphs of the relative frequency polygons) (see Figs. 5i, 6). For HA with EB1, $\beta$-catenin, and PSD-93, relative frequency distributions of pixel intensities were acquired using immunolabeled freshly dissociated neurons and confocal microscopy. In addition, we obtained similar results using either frozen sections or freshly dissociated neurons at both E11-E13 and E18.

Cloning. An E15 chick CG cDNA library was constructed in Hybrizap vector (Stratagene, La Jolla, CA). Full-length human APC cDNA (gift from Dr. Bert Vogelstein, Johns Hopkins University, Baltimore, MD) was used as a probe to screen $5 \times 10^{8}$ clones by standard methods. Six positive clones were isolated, sequenced, and identified as overlapping cDNAs of APC.

Yeast two-hybrid assay. Yeast two-hybrid assays were performed as described previously (Tsen et al., 2000). For the bait construct, chick APC cDNA encoding amino acids 2498-2844 (numbering on the basis of homology to human and mouse sequences) was cloned into pBTM116 vector and expressed as a LexA DNA binding domain fusion in yeast L40 reporter strain. The target was an E15 chick CG cDNA library constructed in pAD-Gal4 vector (excised from Hybrizap).

Coprecipitation assays. In vitro binding assays with recombinant fusion proteins were used to test whether HA-tagged APC dominant-negative peptide prevented the coprecipitation of APC with its targeted binding partners EB1 and PSD-93a. We generated glutathione $S$-transferase (GST) and maltose binding protein (MBP) fusions of chick APC, EB1, and PSD-93a proteins by cloning into pGEX4T-1 and pMalC2 vectors. The fusion proteins were expressed in Escherichia coli BL21-DE3 cells and purified using GST Sepharose (Sigma, St. Louis, MO) or amylose resin 
(New England Biolabs, Beverly, MA). The affinity-isolated recombinant fusion peptide was used to coprecipitate its binding partner from bacterial lysates. The coprecipitation assays were performed in the presence or absence of purified HA-tagged APC dominantnegative peptide. Bound protein complexes were eluted, separated by SDS-PAGE, transferred to nitrocellulose, and probed with antisera to the epitope tag. Specific binding was established by using GST alone (negative control) to precipitate the MBP-tagged protein, and using the MBP tag alone for binding to the recombinant GST protein.

In vivo coimmunoprecipitation of APC with its binding partners $\beta$-catenin and PSD-93 was performed on isolated postsynaptic densities (psds). The psds were isolated by subcellular fractionation using previously described methods (Ehlers, 2003; Parker et al., 2004). Briefly, two E18 chick brains (without the brain stem and cerebellum) were homogenized in homogenization buffer (HB) containing protease inhibitors (4 mM HEPES-NaOH, pH 7.4, $0.32 \mathrm{M}$ sucrose, EDTA free protease inhibitor mixture Complete; Roche) and centrifuged at low speed to remove nuclei and blood cells. The supernatant was diluted with $2 \mathrm{ml}$ of $10 \%$ Percoll-HB, layered on top of a discontinuous $10>20 \%$ Percoll-HB gradient, and centrifuged $33,000 \times$ $g$ in a Beckman L8-80 Ultracentrifuge (Beckman Instruments, Fullerton, CA) using SW-28 rotor. The interface between the two Percoll layers was collected, diluted to $12 \mathrm{ml}$ with $\mathrm{HB}$, and further centrifuged at $20,000 \times g$ for 20 min to pellet the psds. The pellet was resuspended in psd buffer (40 mM HEPES-NaOH, $\mathrm{pH}$ 8.1) containing protease inhibitors plus 1 mм EDTA (Complete tablets; Roche), solubilized with $1 \%$ Triton X-100, recentrifuged, and the final pellet resuspended in $200-400 \mu$ l of psd buffer. The isolated psds were stored at $-80^{\circ} \mathrm{C}$ and used in immunoprecipitation assays. The psds were incubated with $2 \mu \mathrm{g}$ of antibody to APC or $\beta$-catenin, and the immune complexes were affinity precipitated with protein-G or protein-A Sepharose beads (for ms and $\mathrm{rb}$ antibodies, respectively). The bound protein complexes were eluted, separated by SDS-PAGE, and analyzed by immunoblotting with the antibody directed against the other protein of the candidate interacting pair (Ikonomov et al., 1998; Conroy et al., 2003). Controls for antibody binding specificity were included.

Retroviral vector-mediated gene transfer. APC-dn construct [amino acids 2500-2844; corresponding to the C-terminus tail of APC that contains the EB1 and PDZ (postsynaptic density-95/Discs large/zona occludens-1) binding domains] (Fearnhead et al., 2001) was generated and coupled to the sequence encoding the hemaglutinin tag (YPYDVPDYA) at its $5^{\prime}$ end by PCR. The APC-dn cDNA was subcloned into the avian-specific retroviral vectors RCASBP of the A and B envelope subgroup types (Homburger and Fekete, 1996). Viral stocks were prepared in DF1 chicken fibroblast cells (American Type Culture Collection, Manassas, VA). CGs were infected in ovo as described previously (Williams et al., 1998) and sampled 1-2 weeks later.

Electron microscopy. E18 CGs were processed for ultrastructural localization of APC relative to synapses using horseradish peroxidase for detection using previously described methods (Williams et al., 1998).

Surface $\alpha 3-n A C h R$ binding assay. Surface levels of $\alpha 3-\mathrm{nAChRs}$ were assayed by saturable specific binding of $\mathrm{mAb} 35$, followed by biotinylated anti-rat antibody and ${ }^{125}$ I-labeled strepavidin to freshly dissociated, in-
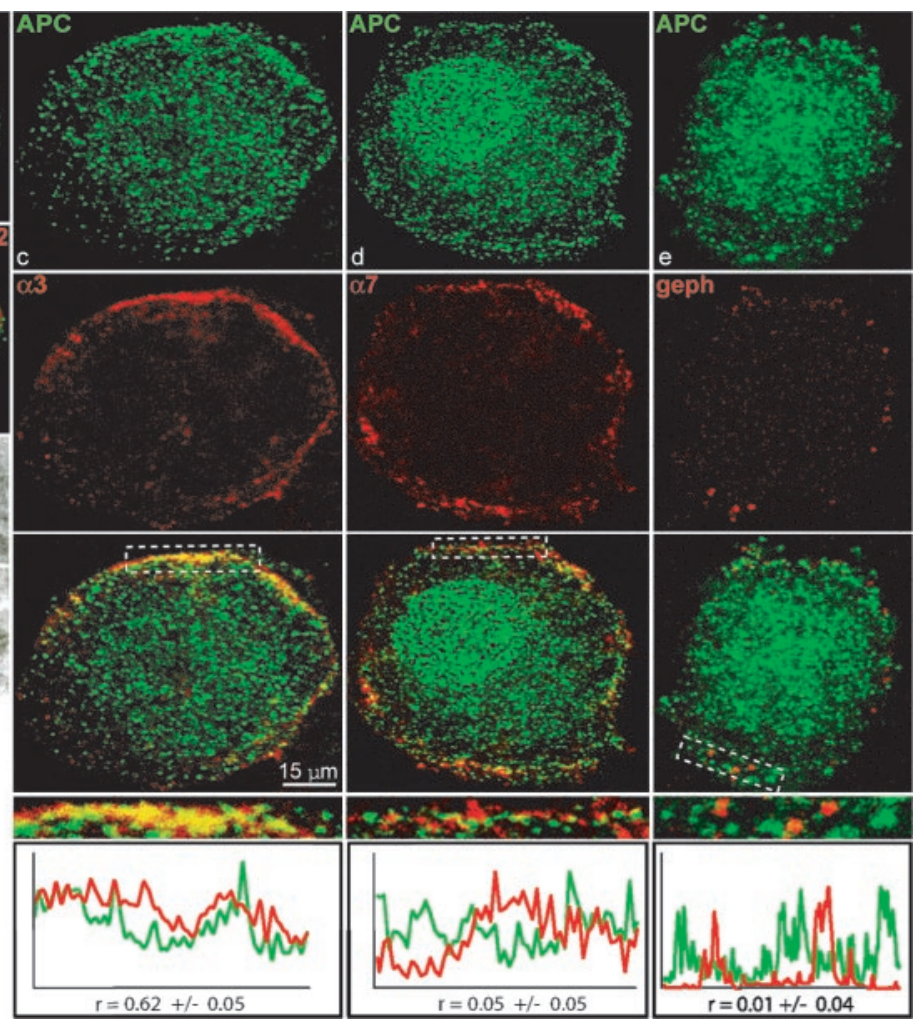

Figure 1. APC localization relative to synapses and the different neurotransmitter receptor types on the CG neuron surface. , 6'-diamidino-2-phenylindole staining (data not shown). $b$, Double-labeled neuron showing that APC surface clusters are

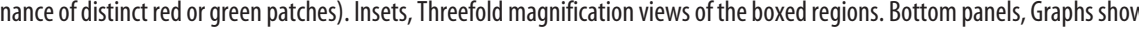
4-6 neurons). $f, g$, Electron micrographs show that APC is localized at the postsynaptic density ( $f$ ) (APC detected by horseradish peroxidase); unlabeled control (ctl) synapse is included for comparison $(g)$.

tact neurons from either two retroviral infected CGs or uninfected control CGs as we have done previously (Williams et al., 1998).

Quantitative reverse transcription-PCR. To compare $\alpha 3$ subunit mRNA levels in individual APC-dn infected CGs versus uninfected control CGs at matched ages, total RNA was isolated, and quantitative reverse transcription (RT)-PCR was performed using $\alpha 3$-specific primers and mutated internal standards as described previously (Levey et al., 1995).

\section{Results}

APC localizes to nicotinic cholinergic, but not glycinergic, postsynaptic sites in chick CG neurons

Our immunofluorescent confocal microscopy studies showed that APC is expressed in chick CG neurons. We used two different anti-APC antibodies that recognize distinct $\mathrm{N}$-terminal and $\mathrm{C}$ terminal epitopes. APC was concentrated at or near the plasma membrane in discrete patches that extended along a portion of the neuron surface (Fig. 1a). APC was also localized in small clusters throughout the cytoplasm and nucleus, as previously described for other cell types (Bienz, 2002). APC surface patches were juxtaposed to presynaptic terminals on the basis of their partial overlap with synaptic vesicle clusters (Fig. 1b). APC strongly colocalized with $\alpha 3$-nAChR surface clusters, as indicated by the predominance of yellow fluorescent patches on the neu- 
rons (Fig. 1c). Fluorescence staining intensities of APC and $\alpha 3$ nAChR surface clusters covaried and strongly correlated with each other [Pearson's correlation coefficient $(r)=0.62 \pm 0.05 ; r$ can range from -1.0 to +1.0 ] (Fig. $1 c$, bottom panel). In contrast, little colocalization was observed for APC with either clusters of $\alpha 7$-nAChRs or GlyRs (detected by staining for the GlyR directly associated protein gephyrin), as indicated by the predominance of distinct red or green patches (Fig. 1d,e). Staining intensities of APC and $\alpha 7-\mathrm{nAChR}$ and GlyR/gephyrin surface clusters did not covary $(r=0.05 \pm 0.05$ and $0.01 \pm 0.04$, respectively) (Fig. $1 d$,e, bottom panels). In addition, we performed 10 separate double-labeling experiments and examined randomly selected neurons by confocal or epifluorescence microscopy. All 90 neurons examined for APC and $\alpha 3$-nAChR labeling showed strong colocalization at the surface membrane. Ultrastructural analysis demonstrated at high resolution that APC is concentrated at the postsynaptic density (Fig. $1 f$ ).

In contrast to the extensive overlap with $\alpha 3$-nAChRs, weak colocalization was observed between APC with $\alpha 7-n$ AChRs and GlyRs: only 29\% (23 of 80) and 15\% (8 of 53) of neurons had any overlap, respectively (a few small punctae of yellow fluorescence). Given that perisynaptic $\alpha 7-n A C h R s$ and synaptic GlyRs are localized in close vicinity to $\alpha 3-\mathrm{nAChR}$-rich postsynaptic membrane regions, some small amount of overlap with APC staining would be expected. Similar to our findings for APC, there is precedence for other proteins showing differential colocalization with the diverse receptor types despite their physical proximity to one another in the surface membrane. For example, PSD-93 and PSD-95 colocalize with $\alpha 3$-nAChRs but not $\alpha 7-n A C h R s$, and gephyrin colocalizes with GlyRs but not $\alpha 3$-nAChRs on CG neurons as detected by immunolabeling with confocal microscopy (Tsen et al., 2000; Conroy et al., 2003). Overall, the colocalization studies showed that APC was selectively enriched at nicotinic cholinergic postsynaptic sites.

Full-length APC and several binding partners are expressed in the $C G$ and interact in vitro

Microtubule end binding protein EB1, $\beta$-catenin, and PSD-95 protein family members are known to interact with APC in noncholinergic cells (Fearnhead et al., 2001). We tested for the presence of these APC binding partners in chick CG neurons. Although a truncated APC isoform that lacks the EB1 and PSD-95 protein family binding domains has been reported (Nakagawa et al., 1998), we found that full-length APC is the dominant isoform expressed in the chick CG by library screening. All six isolated APC clones were overlapping cDNAs of a single isoform that contained the consensus EB1 and PDZ binding domains in the $\mathrm{C}$ terminus. Next, we identified PSD-93 as the PSD-95 protein family member that interacts with APC in the CG by yeast two-hybrid screens of our CG cDNA library using the chick APC C-terminal fragment (amino acids 2498-2844) as bait. The screens identified EB1 and PSD-93 as APC binding partners in the CG. We obtained eight EB1 and three PSD-93 cDNA clones as positives and confirmed their interaction with the chick APC C-terminal fragment by coprecipitation in vitro using recombinant peptides (data not shown). The interacting PSD-93 protein is a short isoform (PSD93a) that lacks the Src homology 3 and guanylate kinase domains and has been shown previously to be expressed in the CG (Conroy et al., 2003).

\section{$A P C$ and its binding partners form a complex in the postsynaptic} density in vivo

We tested for APC interactions with its binding partners at neuronal synapses by coimmunoprecipitation from psds. The psds

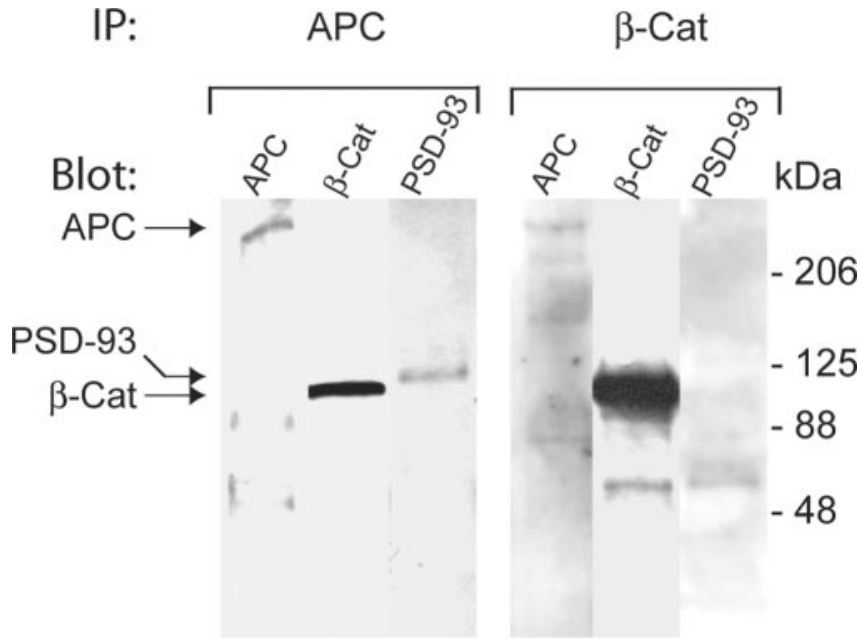

Figure 2. $A P C, \beta$-catenin ( $\beta$-Cat), and PSD-93 form a complex in the postsynaptic density in vivo. The psds were isolated from E18 chick brain (without the cerebellum and brainstem). The psd preparation was immunoprecipitated (IP) with anti-APC antibody, and the immunoprecipitate was probed with antibodies that recognize $\beta$-catenin and PSD-93. In addition, the psds were immunoprecipitated with anti- $\beta$-catenin antibody and probed with antibodies that recognize APC and PSD-93. APC coprecipitated both $\beta$-catenin and PSD-93, whereas $\beta$-catenin coprecipitated APC, but not PSD-93, from the psds. In addition, $\beta$-catenin is more abundant than APC in the coprecipitates, likely because of multiple $\beta$-catenin molecules that bind to one APC molecule via the 15 amino acid and 20 amino acid repeat domains (Rubinfeld et al., 1995; Fearnhead et al., 2001).

were isolated from E18 chick brain by subcellular fractionation (Ehlers, 2003; Parker et al., 2004). We found that APC coprecipitates both $\beta$-catenin and PSD-93, whereas $\beta$-catenin coprecipitates APC, but not PSD-93, from the psds (Fig. 2). These findings confirm a previous report that $\beta$-catenin does not bind directly to PSD-93 (Honjo et al., 2000). Thus, although cytoplasmic $\beta$-catenin, which binds to APC, is targeted for rapid degradation in the Wnt pathway, our data indicate that APC, $\beta$-catenin, and PSD-93 are sequestered and form a complex in the psd in vivo.

\section{APC binding partners localize to CG cholinergic synapses}

We determined by immunolabeling combined with confocal microscopy that PSD-93, $\beta$-catenin, and $\mathrm{N}$-cadherin (the cellular adhesion molecule that binds $\beta$-catenin) were selectively enriched at cholinergic synapses on CG neurons (Fig. $3 a-l$ ). They colocalized with $\alpha 3$-nAChRs and APC at the neuron surface (Fig. $3 a, d, e, h, i, l)$ and were juxtaposed to synaptic vesicle clusters (presynaptic terminals) (data not shown). Fluorescence staining intensities covaried for surface clusters of $\alpha 3-\mathrm{nAChRs}$ and APC with PSD-93, $\beta$-catenin, and $\mathrm{N}$-cadherin and strongly correlated with each other (Fig. 3, table). Thus, although APC and $\mathrm{N}$-cadherin compete for interacting with the same domain of $\beta$-catenin (Hulsken et al., 1994), the three proteins colocalized at nicotinic postsynaptic sites. In contrast, little colocalization was observed for either the neighboring synaptic GlyRs or perisynaptic $\alpha 7-\mathrm{nAChRs}$. Staining intensities of surface clusters of $\alpha 7-\mathrm{nAChR}$ and GlyR/gephyrin and PSD-93, $\beta$-catenin, and $\mathrm{N}$-cadherin were not correlated with each other (Fig. 3, table). In addition, the APC binding partner EB1 showed close proximity to and partial colocalization with $\alpha 3-\mathrm{nAChR}$ and APC surface clusters but did not accumulate much at the surface membrane (Fig. $3 \mathrm{~m}$ ). As expected for a microtubule end binding protein, the bulk of EB1 staining was cytoplasmic. Altogether, our localization studies identify APC, PSD-93, $\beta$-catenin, and N-cadherin as 
components of cholinergic, but not glycinergic, postsynaptic sites in CG neurons.

Overexpressed APC dominant negative caused decreases in $\alpha 3-n A C h R$ surface levels and clusters in vivo

We hypothesized that APC and its binding partners may function in nicotinic cholinergic synapse assembly on the basis of the role of APC surface clusters in capturing EB1-tagged microtubule plus ends at specific surface sites (cadherin/ $\beta$-cateninrich complexes in ectodermal cells) and the synapse organizing functions of PSD-93 and $\beta$-catenin in glutamatergic neurons (Lee et al., 2000; Lu et al., 2001; Sheng and Sala, 2001; Malinow and Malenka, 2002; McGee and Bredt, 2003; Mimori-Kiyosue and Tsukita, 2003; Yu and Malenka, 2003). In particular, we speculate that the interactions of APC with EB1, $\beta$-catenin, and PSD-93 make it a candidate for a role in directing $\alpha 3-\mathrm{nAChR}$ transport to and stabilization at postsynaptic sites. To test this hypothesis, we generated a dominantnegative APC C-terminal fragment (APC$\mathrm{dn})$ to block the interaction of endogenous APC with both EB1 and PSD-93 during synapse formation in CG neurons in vivo. The APC-dn (amino acids 25002844 ) did not contain the $\beta$-catenin binding domain to avoid disrupting the Wnt signaling pathway. In addition, the APC-dn was epitope tagged with HA to distinguish infected cells from uninfected cells by HA immunolabeling. We used retroviral vector-mediated gene transfer to overexpress the APC-dn construct.

Before expression in CG neurons in vivo, we tested the specificity and efficacy of the APC-dn construct. First, we established that HA-tagged APC-dn peptide specifically interacted in vitro with fulllength EB1 and PSD-93a and blocked the binding of APC recombinant fusion protein to these two binding partners by using yeast two-hybrid directed interaction assays and coimmunoprecipitation assays (data not shown). Second, we tested the efficacy of the APC-dn blocking peptide in chick DF1 fibroblasts in vitro. The overexpressed APC-dn led to alterations in the localization of endogenous APC and EB1 (Fig. 4). There were dramatic decreases in endogenous APC and EB1 surface membraneassociated clusters in APC-dn infected fibroblasts compared with uninfected control cells. The APC clusters were distributed in radial arrays throughout the cytoplasm of the APC-dn infected cells (Fig. 4a). This staining pattern suggested localization of APC along microtubules, as expected on the basis of reports that APC binds to and moves along microtubules independent of its interactions with EB1 (Barth et al., 2002; Jimbo et al., 2002). Unfortunately, we were unable to test for changes in endogenous APC and EB1 coprecipitation levels, because the available anti-EB1 antibody did not work in the in vivo coimmunoprecipitation assays as reported previously ( $\mathrm{Su}$ et al., 1995; Berrueta et al., 1999). As an indication of specificity, the overexpressed APC-dn
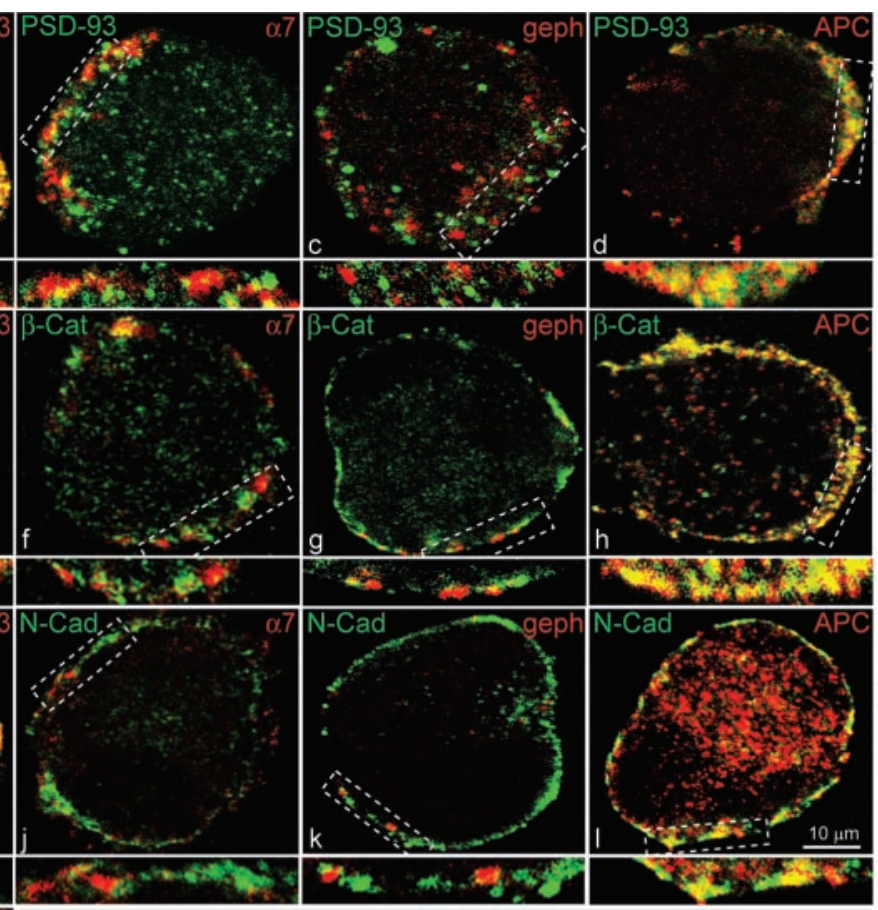

\begin{tabular}{|l|c|c|c|c|}
\hline \multicolumn{5}{|c|}{ Index of colocalization } \\
\hline & $\alpha 3$ & $\alpha 7$ & geph & APC \\
\hline PSD-93 & $0.64^{+/-0.03}$ & $0.13^{+/-0.07}$ & $-0.27^{+/-0} 0.06$ & $0.59^{+/-0} 0.09$ \\
\hline$\beta$-Cat & $0.67^{+/-0.03}$ & $0.09^{+/-0.08}$ & $-0.04^{+/-0.09}$ & $0.87^{+/-0.02}$ \\
\hline N-Cad & $0.69^{+/-0.03}$ & $0.15^{+/-0.06}$ & $-0.17^{+/-0.12}$ & $0.47^{+/-0.09}$ \\
\hline
\end{tabular}

Figure 3. Localization of APC binding partners at nicotinic cholinergic postsynaptic sites. $a, d, e, h, i, l$, Confocal micrographs of double-labeled acutely dissociated E18 CG neurons showing the APC binding partners PSD-93 and $\beta$-catenin ( $\beta$-Cat) and the cellular adhesion molecule $\mathrm{N}$-cadherin ( $\mathrm{N}$-Cad) predominantly colocalized with $\alpha 3$-nAChR $(a, e, i)$ and APC $(d, h, l)$ surface clusters

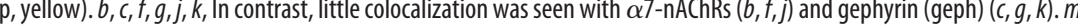
B1 did not accumulate much at the surface membrane. Instead, EB1 labeling was predominantly cytoplasmic. Insets, Twofold correlated with each other for PSD-93, $\beta$-catenin, and N-cadherin with $\alpha 3$-nAChRs and APC but not with $\alpha 7$-nAChRs or GlyRs/ gephyrin ( $n=2-3$ surface areas per cell; $4-6$ neurons).

caused no change in the amounts of APC and $\beta$-catenin that coprecipitated from lysates of retroviral infected versus uninfected control fibroblasts (data not shown). Based on the specificity and efficacy of the APC-dn construct in the in vitro binding assays and DF1 fibroblasts, we overexpressed the blocking peptide in chick CG neurons during synapse formation in vivo.

APC-dn led to specific and dramatic decreases in $\alpha 3$-nAChR surface clusters (Fig. $5 a, b$ ) relative to those on uninfected control CG neurons (Fig. $5 f$ ). In sharp contrast, neighboring $\alpha 7$-nAChR and GlyR clusters were not detectably altered (Fig. $5 d$,e). We found a quantitative correlation between APC-dn expression levels (pixel intensity of HA immunolabeling) and the extent of reductions in $\alpha 3$-nAChR clusters (Fig. $5 h$ ). In particular, the proportion of neurons per frozen ganglion cross-section that had large densely stained $\alpha 3$-nAChR surface clusters was sevenfold lower for heavily APC-dn infected neurons and twofold lower for lightly APC-dn infected neurons relative to the values for control neurons [uninfected neurons within the same CG (internal control) and uninfected age-matched CGs]. In contrast, there were no significant differences in the proportion of neurons with large densely stained GlyR and $\alpha 7$-nAChR surface clusters for APC-dn 

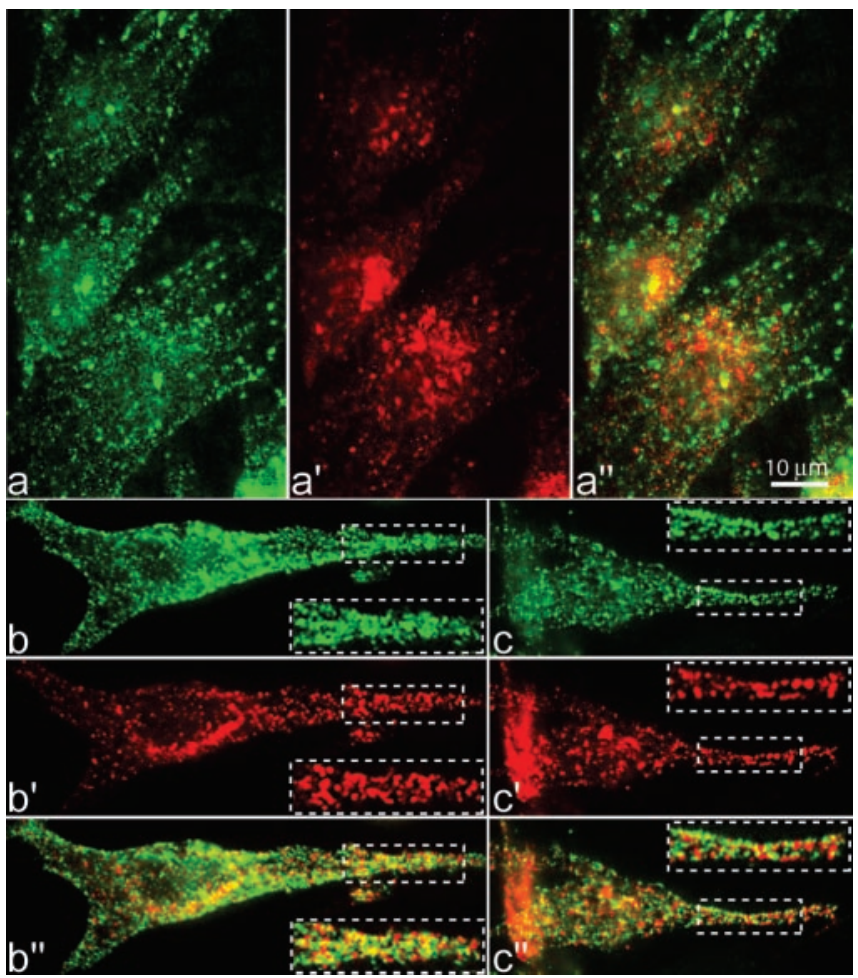

Figure 4. Efficacy of the overexpressed APC dominant-negative construct tested in chick fibroblasts in vitro. $a-c$, Epifluorescence micrographs of double-labeled chick DF1 fibroblasts showing that the overexpressed APC-dn ( $a$ ) led to drastic decreases in endogenous APC (green) and EB1 (red) surface membrane-associated clusters compared with uninfected control fibroblasts $(b, c)$. The APC clusters were distributed in radial arrays throughout the cytoplasm of the APC-dn infected cells (a). To distinguish endogenous APC from the exogenous APC-dn (APC C-terminal peptide fragment), we used the anti-APC antibody that recognizes the N-termina epitope. Insets, Twofold magnification views of boxed regions in control uninfected fibroblasts $(b, c)$

infected neurons versus uninfected control neurons (Fig. 5h). In addition, we also measured the pixel intensities of $\alpha 3-\mathrm{nAChR}$ surface membrane labeling in heavily APC-dn infected versus uninfected control neurons (Fig. 5i). The relative frequency distribution of the pixel intensities shows that there is a substantial decrease in the high fluorescence intensity labeling in the APC-dn expressing neurons. In addition, decreases in $\alpha 3$-nAChR clusters were seen at both early (E7) (data not shown) and late (E13) stages of synapse formation and maturation, suggesting that the clusters never form (as opposed to their forming and not being retained).

To test whether the decrease in $\alpha 3$-nAChR clusters reflected a diffuse distribution or reduced receptor numbers, we measured $\alpha 3$-nAChR surface levels by specific binding of anti-receptor antibody (mAb35), biotinylated secondary antibody, and ${ }^{125} \mathrm{I}-$ strepavidin to freshly dissociated neurons from individual CGs (Williams et al., 1998). There were twofold reductions in the total number of surface $\alpha 3$-nAChRs per APC-dn infected CG relative to uninfected control CG values ( $p<0.01$; Student's $t$ test; $n=$ 35 APC-dn CGs and 38 control CGs from six separate experiments) (data not shown). This analysis underestimates the decrease in surface $\alpha 3-n A C h R s$ because not all neurons in the CG were infected $(20 \pm 3 \%$ of the neurons per CG did not have detectable HA-APC-dn immunolabeling).

The specific changes in $\alpha 3$-nAChRs, but not GlyRs and $\alpha 7$ $n A C h R s$, suggested that the effects of the overexpressed blocking peptide were restricted to the targeted interactions. As additional tests for specificity, APC-dn overexpression caused no significant change in the following: intracellular $\alpha 3$-nAChR levels, representing the biosynthetic pool (Fig. $5 c$ ); $\alpha 3$ subunit mRNA levels (quantitative RT-PCR); and presynaptic terminal morphology (synaptic vesicle immunolabeling) (Fig. 5j). Additionally, there was no change in $\alpha 3$-nAChR surface clusters on retroviral-green fluorescent protein (GFP) infected CG neurons (as a negative control) (Fig. 5k). Overall, the overexpressed APC-dn caused specific changes in surface $\alpha 3$-nAChRs.

To confirm the in vivo efficacy of the dominant negative construct and further define the synapse organizing functions of APC interactions, we looked for changes in surface levels of APC and its binding partners in infected versus uninfected control CG neurons. We found no change in APC or $\beta$-catenin surface levels (frequency distribution of pixel intensities), whereas EB1 and PSD-93 surface membrane-associated labeling was reduced (Fig. 6). Similar to our results, APC interactions with EB1 are not required for APC localizing to specialized surface sites in polarized epithelial cells (Barth et al., 2002). Together, our findings suggest that the overexpressed APC-dn specifically blocked endogenous APC interactions with both EB1 and PSD-93 in CG neurons in vivo. Importantly, the specific decreases in PSD-93 and EB1 surface membrane-associated clusters further suggest that APC interactions are required to localize or selectively retain these two binding partners at nicotinic cholinergic postsynaptic sites.

\section{Discussion}

The major finding reported here is that the tumor-suppressor protein APC is required for high-density accumulations of $\alpha 3$ nAChRs at postsynaptic sites in CG neurons in vivo. This study identifies APC as the first nonreceptor protein to function in localizing nAChRs at neuronal synapses. We identify EB1, $\beta$-catenin, and PSD-93 as APC binding partners in the CG. We show that APC, PSD-93, $\beta$-catenin, and N-cadherin (the cell adhesion molecule that binds $\beta$-catenin) are selectively enriched at cholinergic synapses; they colocalize with surface clusters of $\alpha 3$ nAChRs and one another and are juxtaposed to synaptic vesicle clusters on CG neurons. In contrast, little colocalization was observed for either the neighboring synaptic GlyRs or perisynaptic $\alpha 7$-nAChRs. In addition, the microtubule end-binding protein EB1 shows close proximity to and partial overlap with surface clusters of $\alpha 3$-nAChRs and APC. Importantly, in vivo overexpression of an APC dominant-negative peptide causes dramatic decreases in $\alpha 3$-nAChR surface levels and clusters but not of GlyRs or $\alpha 7$-nAChRs. In addition, the overexpressed APC-dn also leads to reductions in PSD-93 and EB1 surface membraneassociated clusters. These results demonstrate that APC plays a key role in the assembly of excitatory cholinergic, but not inhibitory glycinergic, postsynaptic specializations in CG neurons. This work provides new insights into molecular interactions that direct the formation of neuronal nicotinic synapses in vivo and defines a neural function for APC

Blocking the interactions of APC with both EB1 and PSD-93 dramatically decreases $\alpha 3-\mathrm{nAChR}$ surface clusters. However, new studies indicate that PSD-93 is not required for $\alpha 3-\mathrm{nAChR}$ clustering at neuronal synapses (Conroy et al., 2003; Parker et al., 2004). Together, the data suggest that the interactions of APC with EB1, but not with PSD-93, target $\alpha 3$-nAChRs to postsynaptic sites in neurons.

We speculate that the interactions of APC with EB1 direct $\alpha 3$-nAChR surface delivery (microtubule-mediated transport) or stabilization at postsynaptic sites by regulating the micro- 
tubule cytoskeleton. In our model, EB1 tags the plus ends of a subset of microtubules, and APC accumulates at synapses marked by $\beta$-catenin/N-cadherin complexes. APC directs $\alpha 3$-nAChR delivery to the synapse by capturing EB1-tagged microtubule plus ends and thereby positioning a microtubulebased transport pathway in close proximity to $\beta$-catenin/N-cadherin-marked postsynaptic regions. In addition, APC and EB1 interactions may be required for stabilizing $\alpha 3-n A C h R s$ at postsynaptic sites by anchoring microtubules and thereby regulating microtubule-cortical actin cytoskeletal interactions. Moreover, these two APC functions may not be mutually exclusive. Our model is based on the roles of APC and EB1 in polarized epithelial cells (Dikovskaya et al., 2001; Lu et al., 2001; Bienz, 2002). In these cells, APC accumulates at specific surface sites and directs the capture of EB1tagged microtubules (to the $\beta$-catenin/ cadherin-rich adherens junctions in Drosophila ectodermal cells) (Lee et al., 2000; Lu et al., 2001; McCartney et al., 2001; Barth el al., 2002; Mimori-Kiyosue and Tsukita, 2003). Thus, APC and EB1 interactions anchor microtubule plus ends at precise positions of the cell surface. A key role for APC in tethering microtubules is also suggested by studies that show heterozygous APC mutant mice have substantially fewer microtubule arrays anchored to the specialized basal membrane of polarized epithelial supporting cells (Mogensen et al., 2002). All together, the data suggest that the interactions of APC with EB1 may direct high-density accumulations of $\alpha 3$-nAChRs at postsynaptic sites in vivo.

PSD-93 is a member of the PSD-95related protein family and a well characterized component of the glutamatergic postsynaptic complex. PSD-93 interacts with and links cytoskeletal, signaling, and transmembrane proteins (Brenman et al., 1996; Kim et al., 1996; McGee et al., 2001; Sheng and Sala, 2001; Garner et al., 2002; Malinow and Malenka, 2002; McGee and Bredt, 2003). Surprisingly, we and others found that PSD-93 is also present at neuronal nicotinic cholinergic postsynaptic sites (Conroy et al., 2003; Parker et al., 2004; our study). However, studies of PSD-93 function using knock-out mice or in vitro models have not yet elucidated the role of PSD-93 in cholinergic synapse formation. The studies show that PSD-93 is not required for cholinergic synapses and $\alpha 3$-nAChR clusters to form or for normal levels of functional nAChRs on autonomic neurons (Conroy et al., 2003; Parker et al., 2004). However, loss of synaptic PSD-93 and PSD-95 in vitro reduces spontaneous EPSC frequency (Conroy et al., 2003). The decreased synaptic activity suggests that PSD-93 may be required to organize a complex that signals retrogradely to the presynaptic terminal and thereby regulates synaptic function (Dean et al., 2003; Prange and El-Husseini, 2003). We identified PSD-93 as an APC binding partner in the CG. Importantly, we show that the
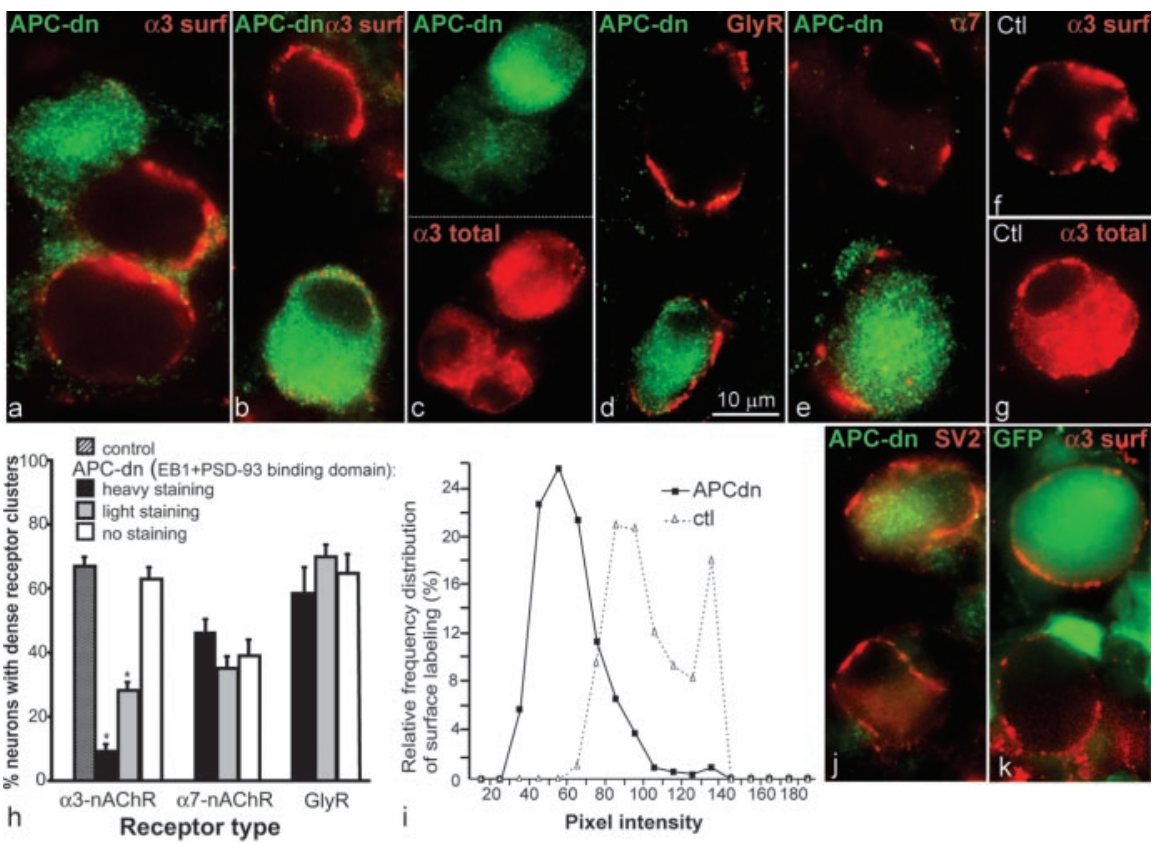

Figure 5. In vivo overexpression of an APC dominant-negative peptide reduced surface clusters (surf) of $\alpha 3$-nAChRs, but not of $\alpha 7$-nAChRs or GlyRs, on CG neurons. Epifluorescence micrographs show double-labeled E11-E13 CG frozen sections. $a-C, \alpha 3-$ $\mathrm{AAChR}(a, b$; red) surface clusters were dramatically reduced in neurons overexpressing the HA-tagged APC-dn (green) compared (a) CGs, individual neurons were classified into three groups on the basis of APC-dn expression levels as judged by the pixel intensity immunolabeling: heavy, light, or not detectable. Cells considered to be heavily labeled had pixel intensities ranging from 105

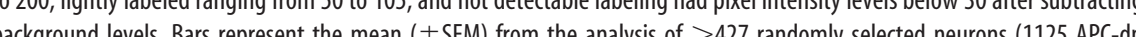
infected neurons for $\alpha 3$-nAChR clusters) from three or more embryos. The asterisk indicates significant difference from controls $(p<0.001$; ANOVA). $i$, Graph showing substantial decreases in the pixel intensity of $\alpha 3-\mathrm{nAChR}$ surface labeling on heavily APC-dn infected neurons versus uninfected control neurons at matched ages. Pixel intensities were measured along randomly were binned into incremental groups of 10 pixel intensity steps (from 0 to 9,10 to $19 \ldots$, up to saturation). We then calculated the percentage of pixels that belonged to each pixel intensity category and plotted the data as relative frequency polygons.

overexpressed APC-dn led to decreases in PSD-93 surface clusters in CG neurons, suggesting that APC interactions are required to localize or selectively retain PSD-93 at nicotinic postsynaptic sites in vivo.

Similar to our findings in neurons in vivo, recent work in muscle shows that APC localizes to the neuromuscular junction and is required for $\mathrm{nAChR}$ clustering induced by agrin on myotubes in vitro (Wang et al., 2003). Interestingly, APC binds directly to the $\beta 1$-subunit of the muscle-type nAChRs. In contrast, we found that neither APC nor its binding partners EB1 and PSD-93a directly bind to $\alpha 3$ (yeast two-hybrid-directed interaction assays and in vitro coimmunoprecipitation assays; data not shown). We focused on $\alpha 3$ because our previous studies showed that this subunit is essential to target heteromeric nAChR channels to synapses in CG neurons in vivo (Williams et al., 1998). However, there is no significant sequence identity between the region of $\beta 1$ that binds to APC and any of the nAChR subunits expressed in CG neurons ( $\alpha 3, \alpha 5, \beta 2, \beta 4$, or $\alpha 7)$. 

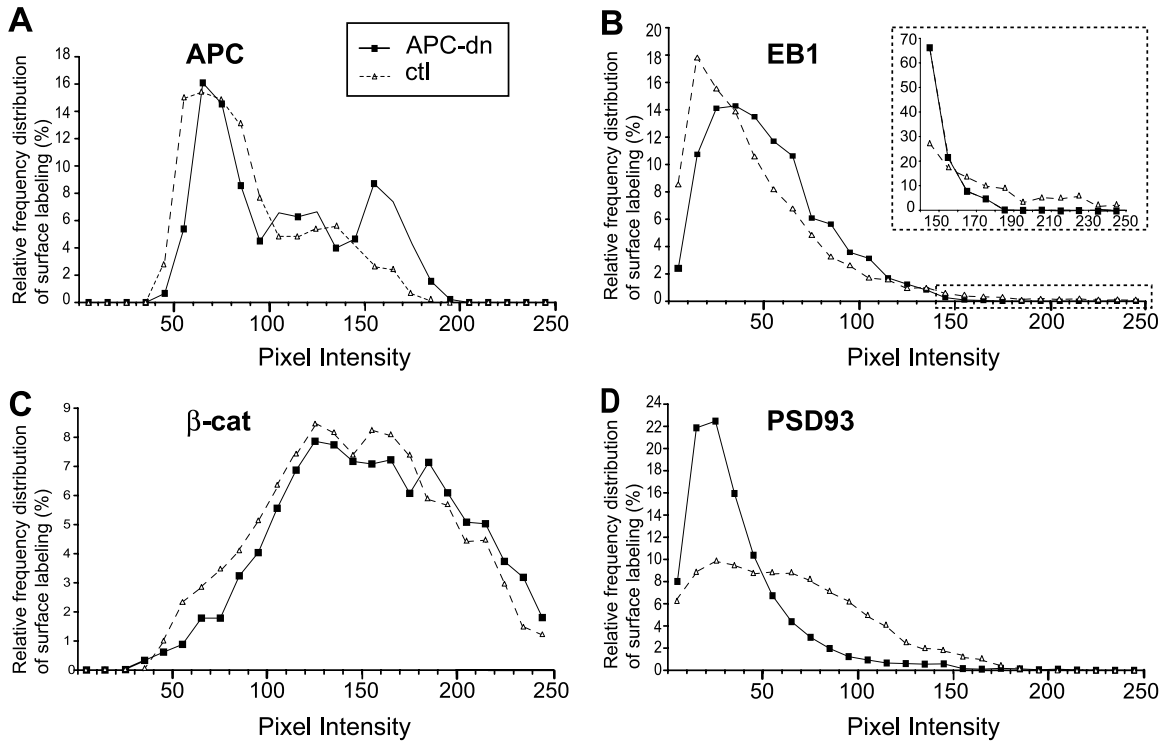

Figure 6. In vivo overexpression of the APC dominant-negative peptide led to decreases in EB1 and PSD-93 surface membraneassociated labeling in CG neurons. $A-D$, The graphs show decreases in the pixel intensity of EB1 $(B)$ and PSD-93 (D) surface membrane-associated labeling in APC-dn infected neurons versus uninfected control CG neurons. Inset, To enhance visualization of the decrease in EB1 surface membrane-associated labeling, we separately calculated and graphed the relative frequency distribution of pixel intensities in the higher range only (pixel intensities from 140 to 255). In contrast, $\operatorname{APC}(A)$ and $\beta$-catenin ( $\beta$-cat) ( $C$ ) surface labeling was not detectably different in infected versus uninfected control C $G$ neurons $(n=3$ or more labeled surface membrane segments per neuron; $4-6$ neurons for each immunolabel).

Consistent with our model that APC functions with other proteins to localize $\alpha 3-\mathrm{nAChRs}$ to synapses, $\alpha 3$-nAChRs and PSD-93 coprecipitate in a complex in vivo from mammalian and avian autonomic ganglia (Conroy et al., 2003; Parker et al., 2004). However, the proteins do not interact directly in the in vitro binding assays, similar to our findings (Conroy et al., 2003). None of the nAChR subunits have an apparent consensus PDZbinding motif. Thus, an adapter-like protein may be required to link $\alpha 3$-nAChRs to PSD-93 or APC. Precedence exists for an intermediate protein (Stargazin) linking neurotransmitter receptors (AMPA receptors) and PSD-95 (Chen et al., 2000; Schnell et al., 2002).

The importance of the role of APC in the vertebrate nervous system is highlighted by the correlation of APC gene deletions with various forms of human mental retardation (Raedle et al., 2001). However, the neural function of APC was previously undefined. We show here that APC interactions with its binding partners are required for high-density accumulations of $\alpha 3$ nAChRs at CG synapses in vivo and thereby define a neural function for APC. In addition, APC is the first overlapping component in neurons and muscle identified to function in nicotinic cholinergic synapse formation. Moreover, APC is also concentrated at glutamatergic synapses in hippocampal neurons (Matsumine et al., 1996). The APC binding partner PSD-93 has organizing functions at glutamatergic and nicotinic cholinergic synapses, whereas both proteins are excluded from inhibitory neuronal synapses (Sheng and Sala, 2001; Conroy et al., 2003; McGee and Bredt, 2003; Parker et al., 2004; our study). In summary, accumulating evidence indicates that APC and its interacting proteins may be core organizers of excitatory, but not inhibitory, synapses in the vertebrate nervous system.

\section{References}

Barth AI, Siemers KA, Nelson WJ (2002) Dissecting interactions between $\mathrm{EB} 1$, microtubules and APC in cortical clusters at the plasma membrane. J Cell Sci 115:1583-1590.
Berrueta L, Tirnauer JS, Schuyler SC, Pellman D, Bierer BE (1999) The APC-associated protein EB1 associates with components of the dynactin complex and cytoplasmic dynein intermediate chain. Curr Biol 9:425-428.

Bienz M (2002) The subcellular destinations of APC proteins. Nat Rev Mol Cell Biol 3:328-338.

Brakeman JS, Gu SH, Wang XB, Dolin G, Baraban JM (1999) Neuronal localization of the Adenomatous polyposis coli tumor suppressor protein. Neuroscience 91:661-672.

Brenman JE, Christopherson KS, Craven SE, McGee AW, Bredt DS (1996) Cloning and characterization of postsynaptic density 93 , a nitric oxide synthase interacting protein. J Neurosci 16:7407-7415.

Chen L, Chetkovich DM, Petralia RS, Sweeney NT, Kawasaki Y, Wenthold RJ, Bredt DS, Nicoll RA (2000) Stargazin regulates synaptic targeting of AMPA receptors by two distinct mechanisms. Nature 408:936-943.

Conroy W, Liu Z, Nai Q, Coggan J, Berg DK (2003) PDZ-containing proteins provide a functional postsynaptic scaffold for nicotinic receptors in neurons. Neuron 38:759-771.

Dean C, Scholl FG, Choih J, DeMaria S, Berger J, Isacoff E, Scheiffele P (2003) Neurexin mediates the assembly of presynaptic terminals. Nat Neurosci 6:708-716.

Dikovskaya D, Zumbrunn J, Penman GA, Nathke IS (2001) The adenomatous polyposis coli protein: in the limelight out at the edge. Trends Cell Biol 9:378-384.

Ehlers MD (2003) Activity level controls postsynaptic composition and signaling via the ubiquitin-proteasome system. Nat Neurosci 6:231-242.

Fearnhead NS, Britton MP, Bodmer WF (2001) The ABC of APC. Hum Mol Genet 10:721-733.

Garner CC, Zhai RG, Gundelfinger ED, Ziv NE (2002) Molecular mechanisms of CNS synaptogenesis. Trends Neurosci 25:243-251.

Homburger SA, Fekete DM (1996) High efficiency gene transfer into the embryonic chicken CNS using B-subgroup retroviruses. Dev Dyn 206:112-120.

Honjo Y, Nakagawa S, Takeichi M (2000) Blockade of cadherin-6B activity perturbs the distribution of PSD-95 family proteins in retinal neurones. Genes Cells 5:309-318.

Hulsken J, Birchmeier W, Behrens J (1994) E-cadherin and APC compete for the interaction with beta-catenin and the cytoskeleton. J Cell Biol 127:2061-2069.

Ikonomov O, Kulesa M, Shisheva A, Jacob MH (1998) Innervation and target tissue interactions induce Rab-GDP dissociation inhibitor (GDI) expression during peripheral synapse formation in developing chick ciliary ganglion neurons in situ. J Neurosci 18:6331-6339.

Jacob MH, Berg DK (1983) The ultrastructural localization of $\alpha$-bungarotoxin binding sites in relation to synapses on chick ciliary ganglion neurons. J Neurosci 3:260-271.

Jacob MH, Lindstrom JM, Berg DK (1986) Surface and intracellular distribution of a putative neuronal nicotinic acetylcholine receptor. J Cell Biol 103:205-214.

Jimbo T, Kawasaki Y, Koyama R, Sato R, Takada S, Haraguchi K, Akiyama T (2002) Identification of a link between the tumor suppressor APC and the kinesin superfamily. Nat Cell Biol 4:323-327.

Kim E, Cho KO, Rothschild A, Sheng M (1996) Heteromultimerization and NMDA receptor clustering activity of Chapsyn-110, a member of the PSD-95 family of proteins. Neuron 17:103-113.

Lee L, Tirnauer JS, Li J, Schuyler SC, Liu JY, Pellman D (2000) Positioning of the mitotic spindle by a cortical-microtubule capture mechanism. Science 287:2260-2262.

Levey MS, Brumwell CL, Dryer SE, Jacob MH (1995) Innervation and target tissue interactions differentially regulate acetylcholine receptor subunit transcript levels in developing neurons in situ. Neuron 14:153-162.

Livingston DM (2001) Cancer: chromosome defects in the colon. Nature 410:536-537. 
Loring RH, Zigmond RE (1987) Ultrastructural distribution of 125I-toxin F binding sites on chick ciliary neurons: synaptic localization of a toxin that blocks ganglionic nicotinic receptors. J Neurosci 7:2153-2162.

Lu B, Roegiers F, Jan LY, Jan YN (2001) Adherens junctions inhibit asymmetric division in the Drosophila epithelium. Nature 409:522-525.

Malinow R, Malenka RC (2002) AMPA receptor trafficking and synaptic plasticity. Annu Rev Neurosci 25:103-126.

Matsumine A, Ogai A, Senda T, Okumura N, Satoh K, Baeg GH, Kawahara T, Kobayashi S, Okada M, Toyoshima K, Akiyama T (1996) Binding of APC to the human homolog of the Drosophila discs large tumor suppressor protein. Science 272:1020-1023.

McCartney BM, McEwen DG, Grevengoed E, Maddox P, Bejsovec A, Peifer M (2001) Drosophila APC2 and Armadillo participate in tethering mitotic spindles to cortical actin. Nat Cell Biol 3:933-938.

McGee AW, Bredt DS (2003) Assembly and plasticity of the glutamatergic postsynaptic specialization. Curr Opin Neurobiol 13:111-118.

McGee AW, Topinka JR, Hashimoto K, Petralia RS, Kakizawa S, Kauer F, Aguilera-Moreno A, Wenthold RJ, Kano M, Bredt DS (2001) PSD-93 knock-out mice reveal that neuronal MAGUKs are not required for development or function of parallel fiber synapses in cerebellum. J Neurosci 21:3085-3091.

Migaud M, Charlesworth P, Dempster M, Webster LC, Watabe AM, Makhinson M, He Y,Ramsay MF, Morris RG, Morrison JH, O'Dell TJ, Grant SG (1998) Enhanced long-term potentiation and impaired learning in mice with mutant postsynaptic density-95 protein. Nature 396:433-439.

Mimori-Kiyosue Y, Tsukita S (2003) "Search-and-capture" of microtubules through plus-end-binding proteins (+TIPs). J Biochem (Tokyo) 134:321-326.

Mogensen MM, Tucker JB, Mackie JB, Prescott AR, Nathke IS (2002) The adenomatous polyposis coli protein unambiguously localizes to microtubule plus ends and is involved in establishing parallel arrays of microtubule bundles in highly polarized epithelial cells. J Cell Biol 157:1041-1048.

Nakagawa H, Murata Y, Koyama K, Fujiyama A, Miyoshi Y, Monden M, Akiyama T, Nakamura Y (1998) Identification of a brain-specific APC homologue, APCL, and its interaction with beta-catenin. Cancer Res 58:5176-5181.
Parker MJ, Zhao S, Bredt DS, Sanes JR, Feng G (2004) PSD93 regulates synaptic stability at neuronal cholinergic synapses. J Neurosci 24:378-388.

Prange O, El-Husseini AE (2003) Instructive role for PSD-95 in neuroliginmediated synapse formation. Soc Neurosci Abstracts 783.1.

Raedle J, Friedl W, Engels H, Koenig R, Trojan J, Zeuzem S (2001) A de novo deletion of chromosome $5 \mathrm{q}$ causing familial adenomatous polyposis, dysmorphic features, and mild mental retardation. Am J Gastroenterol 96:3016-3020.

Rubinfeld B, Souza B, Albert I, Munemitsu S, Polakis P (1995) The APC protein and E-cadherin form similar but independent complexes with alpha-catenin, beta-catenin, and plakoglobin. J Biol Chem 270:5549-5555.

Schnell E, Sizemore M, Karimzadegan S, Chen L, Bredt DS, Nicoll RA (2002) Direct interactions between PSD-95 and stargazin control synaptic AMPA receptor number. Proc Natl Acad Sci USA 99:13902-13907.

Sheng M, Sala C (2001) PDZ domains and the organization of supramolecular complexes. Annu Rev Neurosci 24:1-29.

Shoop RD, Martone ME, Yamada N, Ellisman MH, Berg DK (1999) Neuronal acetylcholine receptors with alpha7 subunits are concentrated on somatic spines for synaptic signaling in embryonic chick ciliary ganglia. J Neurosci 19:692-704.

Su LK, Burrell M, Hill DE, Gyuris J, Brent R, Wiltshire R, Trent J, Vogelstein B, Kinzler KW (1995) APC binds to the novel protein EB1. Cancer Res 55:2972-2977.

Tsen G, Williams B, Allaire P, Zhou YD, Ikonomov O, Kondova I, Jacob MH (2000) Receptors with opposing functions are in postsynaptic microdomains under one presynaptic terminal. Nat Neurosci 3:126-132.

Wang J, Jing Z, Zhang L, Zhou G, Braun J, Yao Y, Wang ZZ (2003) Regulation of acetylcholine receptor clustering by the tumor suppressor APC. Nat Neurosci 6:1017-1018.

Williams B, Temburni MK, Levey M, Bertrand S, Bertrand D, Jacob MH (1998) The long internal loop of the $\alpha 3$ subunit targets nAChRs to subdomains within individual synapses on neurons in vivo. Nat Neurosci 1:557-562.

Yanai H, Satoh K, Matsumine A, Akiyama T (2000) The colorectal tumour suppressor APC is present in the NMDA-receptor-PSD-95 complex in the brain. Genes Cells 5:815-822.

Yu X, Malenka RC (2003) Beta-catenin is critical for dendritic morphogenesis. Nat Neurosci 11:1169-1177. 